\title{
Development of a substorms catalog including the MPB observed at Panagjurishte station, Bulgaria
}

\author{
Veneta Guineva ${ }^{l, *}$, Rolf Werner $^{l}$, Andris Lubchich $^{2}$, Atanas Atanassov ${ }^{l}$, Rumiana \\ Bojilova $^{3}$, Lyubomira Raykova ${ }^{l}$, Dimitar Valev ${ }^{l}$, and Irina Despirak ${ }^{2}$ \\ ${ }^{1}$ Space Research and Technology Institute (SRTI), Bulgarian Academy of Sciences, Stara Zagora \\ Department, Bulgaria \\ ${ }^{2}$ Polar Geophysical Institute, Apatity, Russia \\ ${ }^{3}$ National Institute of Geophysics, Geodesy and Geography (NIGGG), Bulgarian Academy of \\ Sciences, Sofia, Bulgaria
}

\begin{abstract}
In this work the first results of the creation of a substorms catalog including the mid-latitude positive bays (MPB) registered at the Bulgarian station Panagjurishte ( $\sim 37^{\circ}$ GMLat, $\sim 97^{\circ}$ GMLon) are presented. MPB index characterizes the mid-latitude effect of substorm, which developed at auroral latitudes, and it is associated with the substorm current wedge. The work went in several lines. First, the catalog design and content were taken. Second, tools of data processing have been developed. Third, substorms during two months, namely January and February 2013 were identified. And finally, data processing was implemented and the characteristics of the positive bays were determined.

The obtained data and their visualizations were put in a convenient folder and file structure, ftp positioned.
\end{abstract}

\section{Introduction}

Substorms are a typical event at auroral latitudes and a substantial source of magnetic activity. It is known, that the magnetic disturbances during substorms are connected to the auroral electrojets development. The dynamics, intensity and location of the electrojets have been investigated still since the 70s of the past century [e.g. 1-3]. Depending on the conditions in the solar wind and the geomagnetic activity, substorm disturbances, characteristic for auroral latitudes $\left(\sim 60^{\circ}\right.$ to $\sim 71^{\circ}$ GMlat), could reach as very high latitudes (latitudes of the polar cap, $>70^{\circ}$ GMlat $)[2,4,5]$, as well as middle $\left(\sim 50^{\circ}\right.$ GMlat $)$ and even low latitudes $\left(<20^{\circ}\right.$ GMlat). After McPherron et al. [1] midlatitudes are from $20^{\circ}$ to $52^{\circ}$. It is to be noted, that substorms at middle and low latitudes are usually observed during magnetic storms, when the equatorial boundary of the auroral oval is shifted as far as $\sim 50^{\circ}$ GMlat [6].

In contrast to the auroral latitudes, where the magnetic substorms are expressed by sharp negative bays in the $\mathrm{X}$ magnetic component, at midlatitudes the magnetic substorms are observed as positive bays (maxima) in the $\mathrm{X}$ component [1]. First, it was assumed, that the

\footnotetext{
*Corresponding author: v guineva@yahoo.com
} 
low latitude return currents from the westward electrojet have given rise to the positive bays [7], later the positive bays were explained as a result of the upwards field-alligned currents $[8,9]$. Subsequently it was found out, that the midlatitude positive bays were connected to the substorm current wedge $(\mathrm{SCW})$ - a current system including the deviation of the magnetospheric tail current along the magnetic field lines to the ionosphere in the dawn side and its return to the magnetosphere through the dusk side [10-12]. The structure of the SCW was studied in order to verify whether this current system is formed by an ensemble of wedglets [13] and it was suggested, that the SCW is basically a globally coherent system.

A special index was developed, the midlatitude positive bay index (MPB) [14-16], as an indicator of the characteristics of the SCW. It can be used as a proxy of the magnetic substorms onset. This index is widespread in the magnetospheric investigations. For example, a connection has been ascertained between the MPB index maxima and the appearance of plasmoids in the magnetospheric tail [17].

The MPB index was computed by data of 35 low- and midlatitude stations, globally distributed $[14,15]$. A correlation between the MPB index and the SuperMag SML index was found out [16]. But data from the INTERMAGNET European stations and of the midlatitude stations of the Scandinavian longitudinal chain NAL-BRZ were not examined in these studies. The MPB index by data of the European midlatitude stations was not computed up to now. To fill up this gap, the European midlatitude data should be processed to obtain the horizontal magnetic power (main field and mean Solar quite magnetic variations removed) and to use them for the MPB index evaluation.

In this work the development of a catalog including the midlatitude positive bays registered at the midlatitude station Panagjurishte, Bulgaria, and the concomitant studies are presented. The catalog data could be useful in the studies of the relation between the appearence of midlatitude positive bays and the solar wind and Interplanetary Magnetic field (IMF) parameters and for the determination of the concrete drivers in the solar wind responsible for the positive bays at midlatitudes.

\section{Structure of the Panagjurishte substorm catalog}

The Panagjurishte substorm catalog was envisaged to be FTP positionned. The planned structure of the catalog is presented schematically in Fig.1. The main part of the catalog consists of data, derived from the Panagjurishte magnetic measurements. A complimentary part including some additional data about the substorm days is planned, as well. The main part comprises every day data (graphics and data files of the processing results) and data about every substorm (some parameters of the magnetic positive bay). The complimentary part (concomitant data) includes information as the $\mathrm{X}$ bay sign convertion latitude, the structures in the solar wind, some geomagnetic indices for the day and the presence or no of a geomagnetic storm.

\section{Processing procedure: calculation of the horizontal power of the magnetic field}

The midlattitude horizontal power of the magnetic field, main field and mean Solar quite magnetic variations (Sq) removed (MPB) is a usefull measure for substorm identification by midlattitude magnetic data. The midlattitude positive bay index can be evaluated by the average of the horizontal power computed for a number of midlattitude stations. 


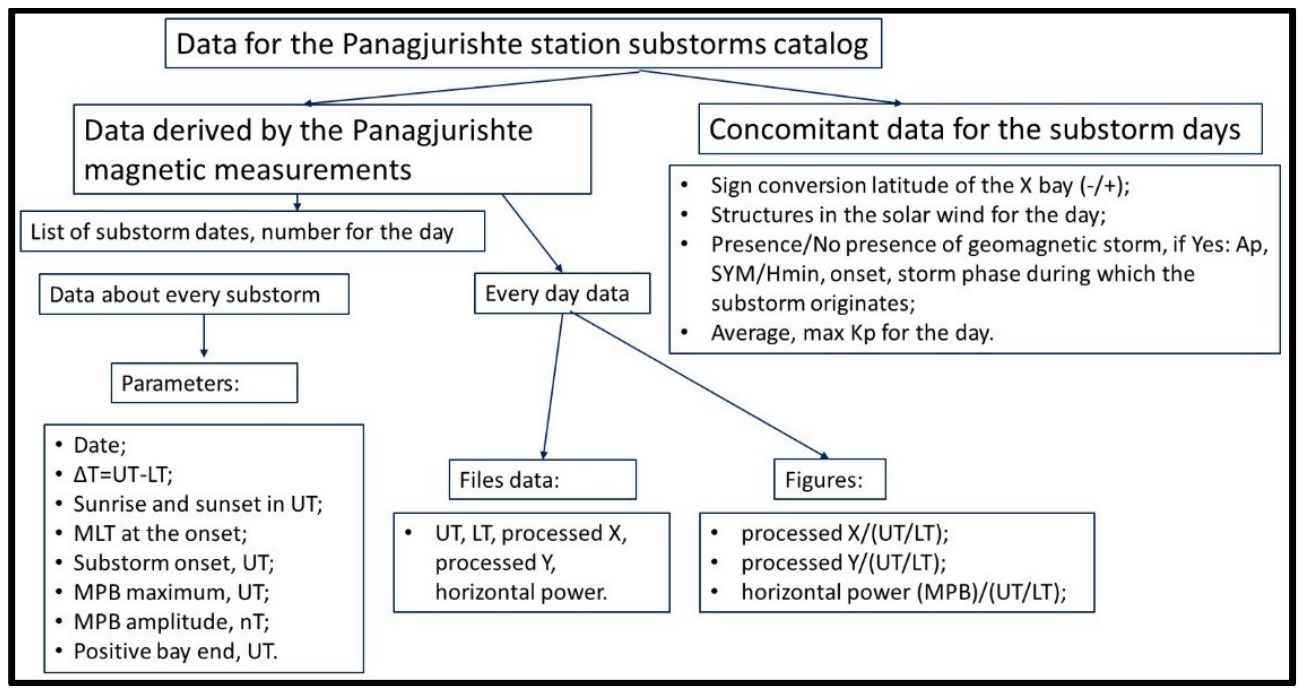

Fig. 1. Scheme of the structure of the Panagjurishte substorm catalog.

\subsection{Data used}

The processing was performed for January and February 2013. Data from the International Real-time Magnetic Observatory Network (Intermagnet) Data Download (intermagnet.org) for the Bulgarian station Panagjurishte (PAG) $\left(42.5^{\circ} \mathrm{N}, 24.2^{\circ} \mathrm{E}, 556 \mathrm{~m}\right.$ altitude; $37^{\circ} \mathrm{GMLat}$, $\sim 97^{\circ}$ GMLon) were used. The one minute mean data for the magnetic field components $X$, $\mathrm{Y}, \mathrm{Z}$ and the total field intensity are given in $\mathrm{nT}$ for moments in UT.

The magnetic field data for 25 successive days centred at the day under consideration were processed. Following the McPherron's algorithm [15] this time interval is sufficient to estimate and remove the mean main magnetic field and the mean field under solar quiet conditions.

\subsection{Preprocessing}

The preprocessing of the observed magnetic data includes procedures for removal of data gaps, for peak detection and for detection and removal of days with very high magnetic disturbances (called for shortness "disturbed days"). Data gapes and peaks are usually randomly distributed and are not concentrated within a day. Therefore as a first step, time series of the whole interval of the considered consecutive 25 days was constructed from the measurements of every magnetic component. Because the data are given for UT, the time series are converted to LT by a corresponding to the geographical longitude data shifting.

\subsubsection{Data gaps removal}

Data gaps have mostly technical reasons as electrical power outage or required maintenance. If no data was registered, then the value 99999 was written in the original data file. In the gapes the values of an additional series are set to a constant many greater than the magnetic variances and so they are well graphically presentable. The interval borders of the data gapes are then easy to found by means of the first differences. The no data values were replaced by the result of the linear interpolation between the last and first measurements outside of the no data interval. In the special case when no data were measured at the beginning or end of 
the 25 days series, the no data were replaced by the nearest observations. For the illustration of the gap and peak detection and removal the data for the substorm on 20.02.2017 were used. In the time series from 08.02.2017 up to 04.03.2017 five gaps were identified. One example is shown in Fig.2.

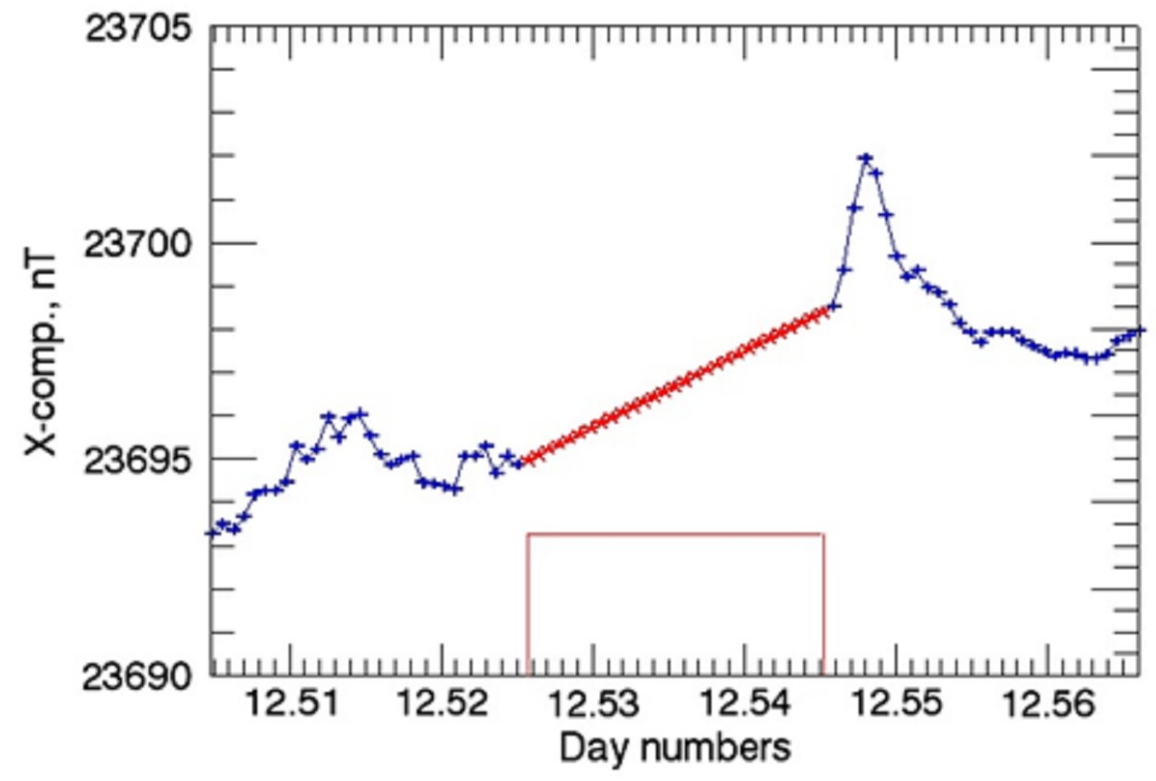

Fig.2. The figure demonstrates the procedure for the gape removal. One gape, observed at the day 13 (20.02.2017) of the time interval is marked by the bar plot. The original data are presented by plusses, connected by lines, and the interpolated data are shown by asterisks, also connected with a line. The sequence of days begins with number one for 08.02.2017.

\subsubsection{Peak detection and removal}

Under a peak here we understand a sudden increase or decrease of the magnetic component data. Such type of peaks are often referred spikes. The reason of spikes can be running engines of cars passing the station periphery. The sudden change can be characterised by the absolute value of the first derivation greater than a given threshold. Based on this assumption a simple and fast procedure of peak detection was developed. The peak borders at the peak base were determined by the first undercut of mean standard deviation of the first derivation at left and right of the peak. The values between the peak borders were replaced in the same manner as for the gape removal, by linear interpolation. The peak detection and removal is organized in a program loop, where in every loop the peak with the maximal first derivation is removed. The detection stops when the first derivations do not exceed the given threshold. An example of detection and removal of a single peak and of a sequence of peaks is presented in Fig. 3.

\subsection{Data processing}

The calculation of the MPB follows in general the algorithm developed by McPherron [15]. 


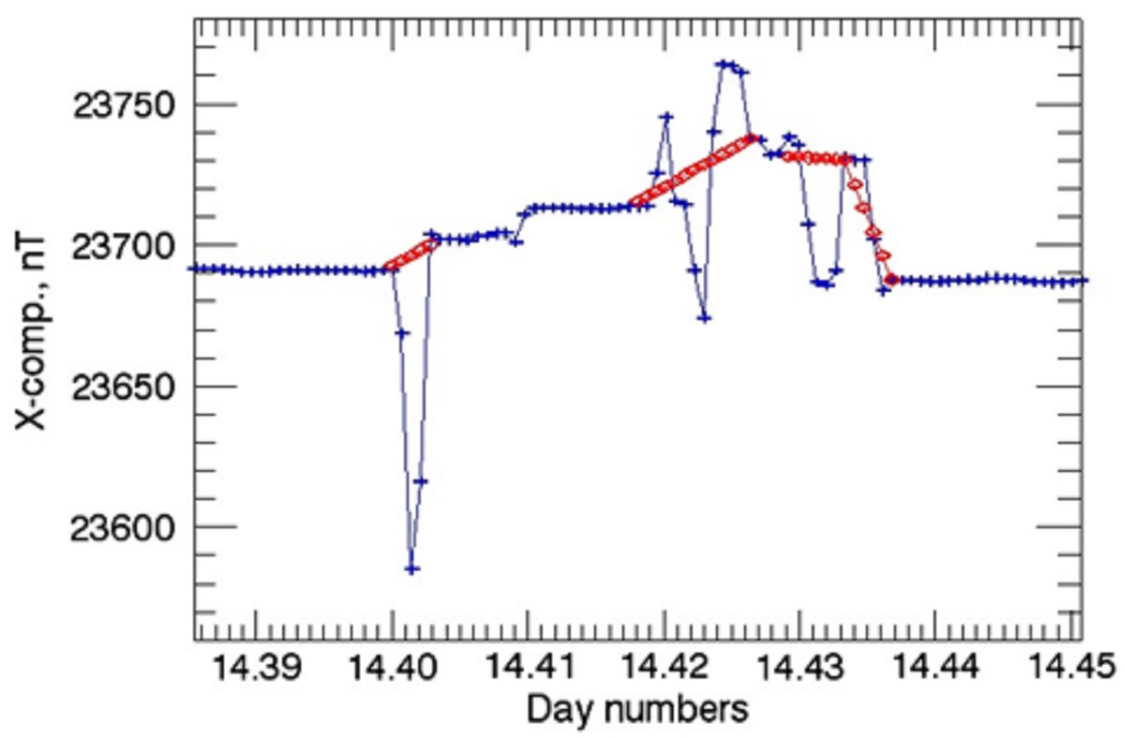

Fig.3. The figure demonstrates how the peak removal is working. The original data are shown by plusses connected by a line. The interpolated data are drawn by diamonds, connected also by a line. In the left side near the day number of 14.40 (22.02.2017) a single peak, detected and removed, is presented. In the right side a sequence of peaks was found and removed by linear interpolation. The sequence of days is the same as in Fig.2.

At first, to eliminate the main magnetic field and the slow changes due to the ring current and partial ring current [15] a base line was constructed by a smoothed spline interpolation through the midnight points (at $24 \mathrm{~h}$ in LT) (see Fig.4). The resulting spline was subtracted from the time series. The remaining series were splitted again in daily sequences but with three additional hours at the beginning and at the end of every day. (For the PAG station data this allows later the easy conversion again to UT). The time segments with the length of 30 hours hereinafter are called extended days. After the time series shift and splitting from the original 25 days (in UT), 23 extended days (in LT) remain. To calculate the mean Solar quite magnetic variations $(\mathrm{Sq})$, extended days with strong magnetic disturbances were removed. The strong variations of the field components during a disturbed day are signalized by their high standard deviation. The standard deviations are calculated for all 23 extended days. The results are considered as random quantities. By the Lilliefors test [18] it was verified, that they are approximately normally distributed. We have determined outliers, meaning disturbed days, applying the Grubbs test, using the one side significance level of 0.12 [19]. The procedure is asymptotic and in average up to two, three disturbed extended days were found. These days were excluded in further calculations. From the remaining days $\mathrm{Sq}$ is determined by averaging of the daily variations of the field components like in the procedure applied in the Chu's algorithm of the MBP calculation [14]. High frequency variations in Sq were smoothed using running mean. Tests to calculate $\mathrm{Sq}$ with the help of Principal component analysis (PCA) were also performed as in the McPherron computations [15]. This procedure is much complicated and slower. Much more, the result is very close to the one obtained by a simple averaging and smoothing. 
X-comp. and smoothed spline, 02.02.2013

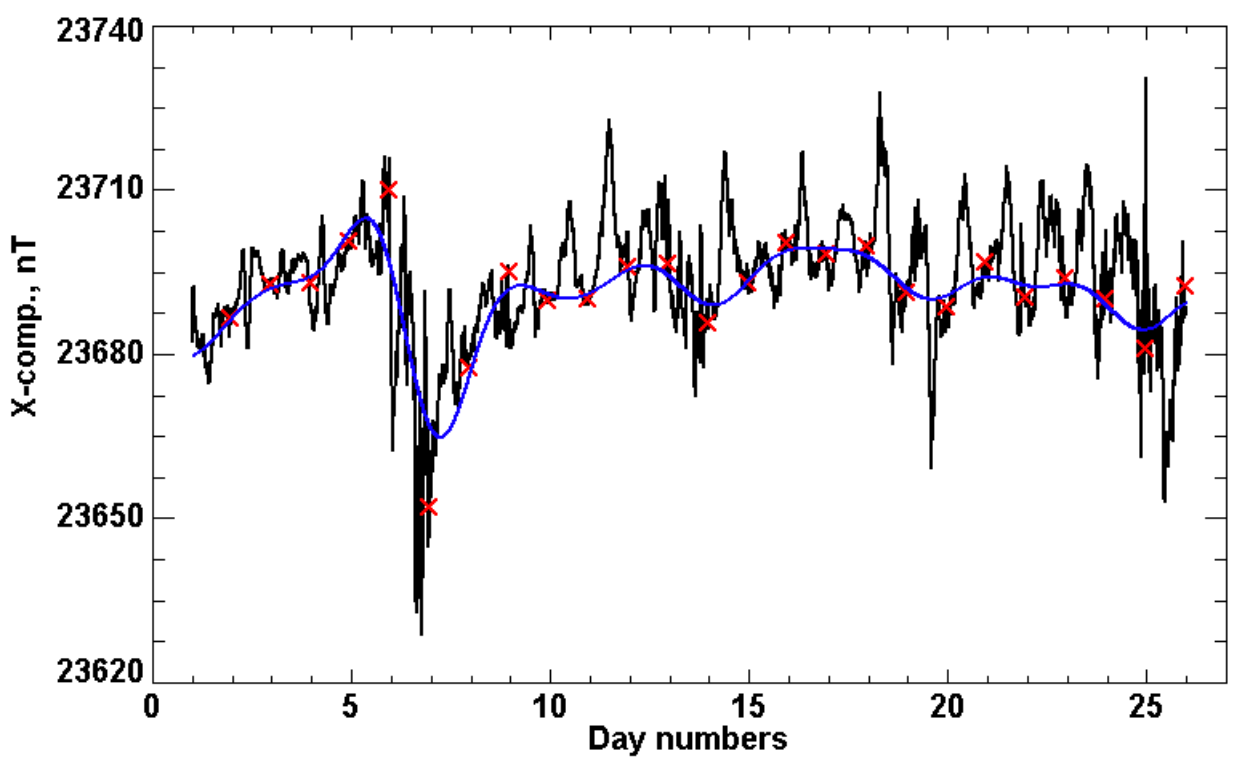

Fig.4. X-component series for 25 days, centered on 02 February 2013. The midnight points (24 LT) are marked by "x" sign. The computed smoothed spline through the midnight points is drawn by a continuous line.

Analogous to the McPherron's method the X and Y components with removed Sq were filtered by a high pass filter to suppress the high frequencies and to keep the low frequency changes. Signals with frequencies longer than about 3 hours were filtered out. Thus only substorm variations remain. In Fig.5, the resulting X (left panel) and Y (right panel) magnetic components for 02 February 2013 are presented. On the $\mathrm{x}$-axis below the figure, the universal time (UT) is indicated, and on the abscissa above the figure - the local time (LT) is shown. The horizontal power of the magnetic perturbations is obtained by the sum of the squared processed X and Y magnetic components (fig6). On the abscissae UT and LT are presented, as in Fig.5.
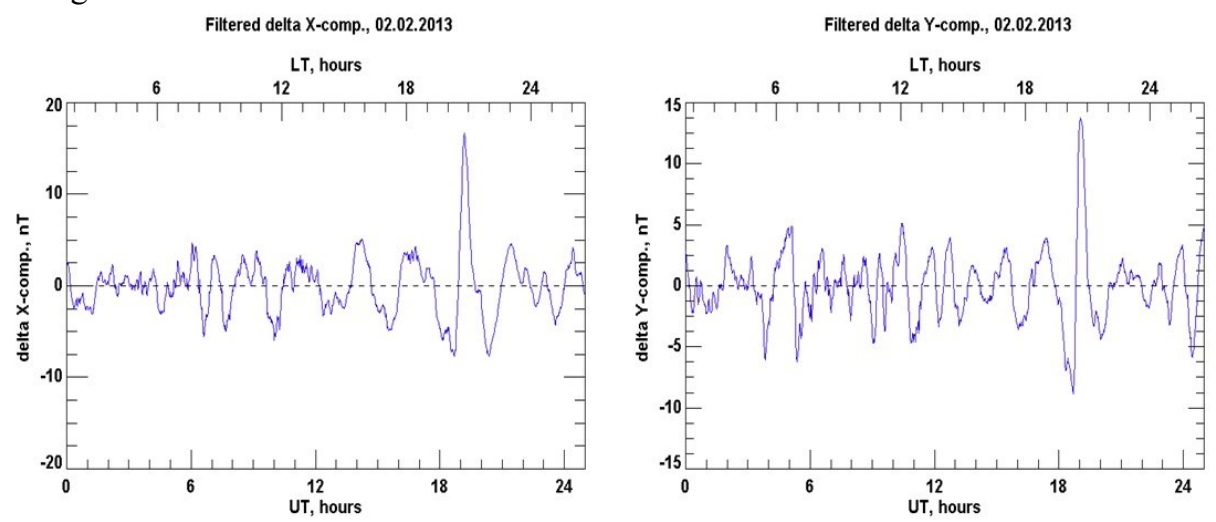

Fig.5. Processed X (left panel) and Y (right panel) components of the magnetic field, measured on 02 February 2013 at the Panagjurishte station. 


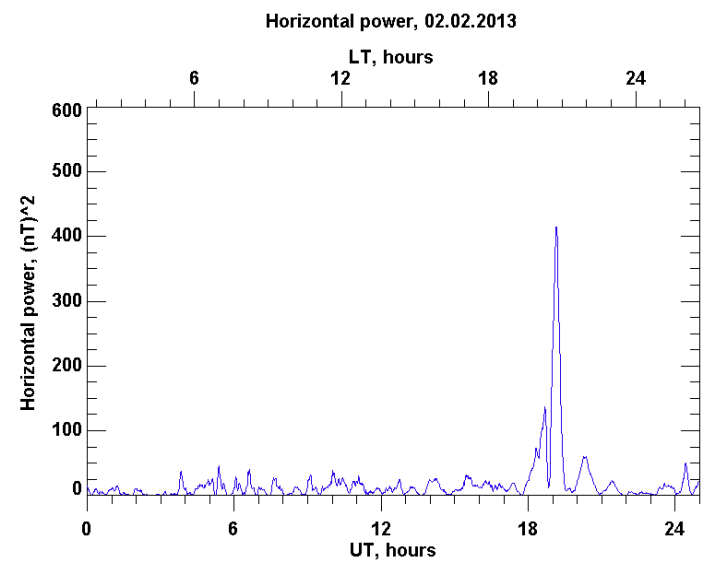

Fig.6. Horizontal power of the magnetic field perturbations on 02 February 2013 at Panagjurishte.

The obtained maximum at about 19:10 UT corresponds to the substorm, observed in the auroral zone, on the IMAGE longitudinal chain PPN-NAL.

\subsection{Every day data included in the catalog}

In the catalog, data files and graphics of some results are included.

The every day files comprise one minute data for the processed $\mathrm{X}$ and $\mathrm{Y}$ magnetic components, and the horizontal power of the magnetic field (main field and mean Solar quite magnetic variations (Sq) removed), called MPB by McPherron [15].

The graphics present the processed $\mathrm{X}$ and $\mathrm{Y}$ components, and the horizontal power of the magnetic field.

The consecutive windows in the Catalog to see the graphics for a given day, are presented in Fig. 7.

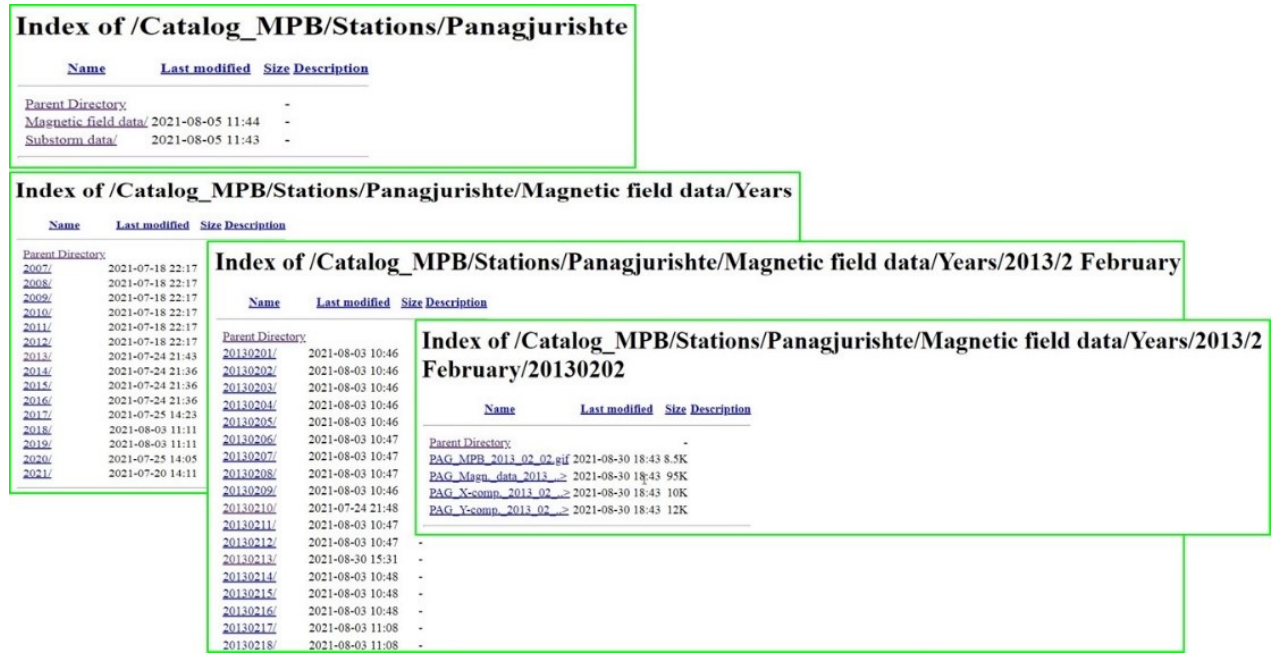

Fig. 7. Consecutive windows to look at the graphics for a given day. 


\section{Detection of the substorms}

We considered all substorms observed during the time interval from January 1 to February 28, 2013 in the auroral zone, i.e. on the IMAGE longitudinal chain (PPN-NAL stations) For each substorm in the auroral zone their midlaltitude displays - positive bays - on the station PAG (Bulgaria) was calculated. We used also data from SuperMAG and INTERMAGNET networks at geomagnetic longitudes near the longitude of the PAG station ( $\sim 97^{\circ}$ GMLon), in the longitudinal band $90^{\circ}-104^{\circ}$ GMLon, to follow the substorms development. For the chosen period 67 substorms were identified, this number includes all observed substorms.

\section{Characteristics of the positive bays}

For the identified substorms, the following parameters of the $\mathrm{X}$ component magnetic positive bay were determined from the PAG station data and included in the catalog (see Fig.8):

- Time of the onset of the positive bay (vertical line (1));

- Time of the positive bay maximum (vertical line (2));

- Amplitude of the X component (difference between the maximum and the minimum at the onset of the $\mathrm{X}$ component) (arrowed line (3));

- Time of the positive bay end (vertical line (4)).

Except these parameters, the following information is also included in the catalog:

- Sunrise and sunset at Panagjurishte for the substorm day;

- Magnetic local time (MLT) at the substorm onset.

In the beginning of every file the date and the difference between local and universal time (LT-UT) for Panagjurishte are written.

The consecutive windows to see the characteristics of the positive bays are similar to these for the day graphs.

(nT)

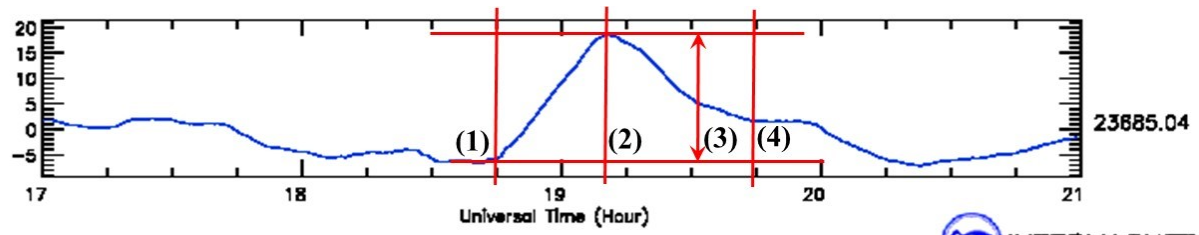

Fig.8. An example of the determination of the MPB parameters. We have chosen an isolated substorm, namely the one at 18:43 UT on 02 February 2013. The way of estimation the MPB parameters is presented on the plot from INTERMAGNET by straight lines, enumerated as follows: (1) time of the substorm onset; (2) time of the positive bay maximum; (3) amplitude of the X-component; (4) time of the positive bay end.

\section{Summary}

A substorms catalog including the horizontal power of the magnetic field, main field and the mean field under solar quiet conditions subtracted, and the positive bays characteristics by data of the midlattitude Bulgarian station Panagjurishte (PAG) is under construction. The catalog is FTP positioned with preliminary address https://magnetic.nuclearmodels.net/Catalog MPB/Stations/Panagjurishte/. The tools indispensable for data preprocessing and processing have been developed. The substorms during January and February 2013 have been identified. The PAG magnetic data for these two months have been processed. The characteristics of the positive bays were determined. The obtained results have been uploaded in the catalog. 
In the future work we intend to process in such a way all one minute magnetic data from Panagjurishte (since 2007) and to complete the substorm catalog.

Such catalog is being made for the first time. This work could be useful for further studies. The processing tools could be used for other European stations data to obtain a dataset. This will allow to implement a complex analysis of the space-temporal characteristics of the magnetic substorms at midlattitudes and auroral latitudes in order to reveal the peculiarities of the spreading of the geomagnetic disturbances to midlattitudes depending on the conditions of the near-Earth space weather.

Acknowledgements. The authors are grateful to the creators of the databases International Real-time Magnetic Observatory Network (Intermagnet) Data Download (intermagnet.org) and IMAGE (http://space.fmi.fi/image/), for the opportunity to use them in this work. We thank the experts from Panagjurishte observatory (NIGGG-BAS) for providing data and support for their processing.

We are grateful to X.N. Chu for the explanations of some processing procedures and the fruitful discussions.

This study was supported by the National Science Fund of Bulgaria (NSFB) (project number КП-06Русия/15) and by the RFBR (project number 20-55-18003Болг_a); the work of I.V. Despirak and A.A. Lubchich carried out within the framework of the RFBR grant No. 20-55-18003_Bulg_a.

\section{References}

1. R.L. McPherron, C.T. Russell, M.G. Kivelson, P.J. Coleman, Jr., Radio Sci., 8(11), 10591076 (1973)

2. J.L. Kisabeth, G. Rostoker, J. Geophys. Res., 79(7), 972-984 (1974)

3. O.A. Troshichev, B.M. Kuznetsov, M.I. Pudovkin, Planet. Space Sci., 22, 1403-1412 (1974)

4. M.I. Pudovkin, O.A. Troshichev, Planet. Space Sci. 20, 1773-1779 (1972)

5. I.V. Despirak, A.A. Lubchich, H.K. Biernat, A.G. Yahnin, Geomagn. Aeron., 48(3), 284292 (2008)

6. C.E. McIlwain, Dordrecht-Holland:D. Reidel, 143-154 (1974)

7. S.-I. Akasofu, S. Chapman, C.-I. Meng, J. Atmos. Terr. Phys., 27, 1275-1305 (1965)

8. S.-I. Akasofu, C.-I. Meng, J. Geophys. Res., 74, 293-313 (1969)

9. C.-I. Meng, S.-I. Akasofu, J. Geophys. Res., 74, 4035-4053 (1969)

10. V.A. Sergeev, R.J. Pellinen, T.I. Pulkkinen, Space Sci. Rev., 75, 551 (1996)

11. X.N. Chu, T.S. Hsu, R.L. McPherron, V. Angelopoulos, Z.Y. Pu, J.J. Weygand, K. Khurana, M. Connors, J. Kissinger, H. Zhang, O. Amm, J. Geophys. Res. Space Phys. 119, 1909-1924 (2014)

12. L. Kepko, R.L. McPherron, O. Amm, S. Apatenkov, W. Baumjohann, J. Birn, M. Lester, R. Nakamura, T.I. Pulkkinen, V. Sergeev, Space Sci. Rev., 190, 1-46 (2014)

13. S. Ohtani, J. Gjerloev, J. Geophys. Res., Space Physics, 125(9) (2020)

14. X. Chu, Configuration and generation of substorm current wedge, Los Angeles: University of California Los Angeles (2015)

15. R.L. McPherron, X. Chu, Space Sci. Rev., 206(1-4), 91-122 (2017)

16. R.L. McPherron, X. Chu, J. Geophys. Res. Space Physics, 123, 2831-2850 (2018)

17. T. Nagai, R. Nakamura, T. Mukai, T. Yamamoto, A. Nishida, S. Kokubun, Adv. Space Res., 20, 961-971 (1997)

18. H.W. Lilliefors, Journal of the American Statistical Association, 62, 399-402 (1967)

19. F.E. Grubbs, G. Beck, Technometrics, 14, 847-857 (1972) 